\title{
Application of Phosphate Solubilizing Bacteria and Its Ecological Effect on Growth and Yield of Winter Maize (Zea Mays L.)
}

\author{
Dr. Rima Taipodia ${ }^{1}$, Dulley Yubbey ${ }^{2}$ \\ ${ }^{I}$ STO O/O Deputy Commissioner, Dibang Valley, Annini, Arunachal Pradesh \\ ${ }^{2}$ Department of Ecology \& Environment, Global open university, Nagaland.
}

\begin{abstract}
A study was conducted on "Application of Phosphate Solubilizing Bacteria \& its Ecological effect on growth and yield of winter maize (Zea mays $L$.)" for two successive winter seasons at Bamin Michi village, Lower Subansiri District Arunachal Pradesh. In split plot design, the experiment was conducted and replicated three times. To the main plots, the three PSB treatments viz, control $\left(T_{1}\right)$, seed inoculation with PSB $\left(T_{2}\right)$ and PSB inoculation along with 5t FYM ha ${ }^{-1}$ were allotted. Due to PSB inoculation along with FYM, the values of all growth parameters (plant height, green leaves, leaf area, LAI and dry matter production plant ${ }^{-1}$ ) recorded higher and remained lower under control. However, on number of days to 50\% silking, maturity and barren plants plot ${ }^{-1}$, the effect of PSB inoculation alone, PSB added with 5t FYM ha $a^{-1}$ and the control found at par to each other though these characters remained higher and lower with PSB added with FYM and the control correspondingly. With the inoculation of PSB added with FYM the values of almost all the yield attributes as well as grain and stover yields were recorded maximum. With the PSB inoculation along with 5t FYM hal ${ }^{-1}$, maximum content of NPK content in grain and stover, as well as their uptake by grain, stover and total uptake by crop were recorded maximum. With inoculation of PSB along with 5t FYM ha ${ }^{-1}$, the available NPKcontent in soil at crop harvest also remained maximum. In respect of protein and carbohydrates in grain, inoculation on PSB along with 5t FYM ha $a^{-1}\left(T_{3}\right)$ found superior
\end{abstract}

Key Words: Hybrid maize; Planting methods; Nutrient management

\section{Introduction}

Maize (Zea mays L.) is an important cereal crop and ranks third in production after rice and wheat in India. Due to higher yield potential, short growing period, high value for food, forage and feed for livestock, poultry and a cheaper source of raw material for agro-based industry, it is increasingly gaining an important position in the cropping system. It has greater nutritional value as it contains about $72 \%$ starch, $10 \%$ protein, 4.8\% oil, $8.5 \%$ fiber, $3 \%$ sugar and 17\% ash (Chaudhry, 1983). Requirement of about $305 \mathrm{mt}$ of food grains is anticipated for 1.4 billion population of India, and the substantive insist for individual food grains has been expected to about $120 \mathrm{mt}$ for rice, $95 \mathrm{mt}$ for wheat, $25 \mathrm{mt}$ for maize and 24-26 mt for pulses by the year 2025 (Tiwari,2001). This decisive state of affairs budding vast strain upon the policy planners for which the response is bigger, diversified as well as sustainable and more rapid grain production.

After the revolution in rice and wheat production, as opined by the Noble laureate, Dr. Norman E. Borlaug, the next few decades will be known as maize era (Rai, 1998).

Phosphate solubilizing bacteria are carrier based preparation in agriculture which may aid in increasing crop productivity by serving in solubilization of insoluble phosphorus, stimulating plant growth by providing hormones, vitamins and other growth factors. In soil $\mathrm{pH}, \mathrm{EC}, \mathrm{O}_{2}, \mathrm{CO}_{2}$ concentration and the presence of organic material, influence the degree of solubilization. As the only sources of organic matter, role of farmyard manure can never be ignored without which soil can never be productive. Farm yard manure (FYM) supplies all the essential nutrients for plants and increase the activity of phosphorus solubilizing bacteria in addition to this.

In view of above mentioned factors, it is desirable to build up cost effective, eco- friendly, sustainable systems, where the supply of phosphorus along with other nutrient to plants be secured. Based on research findings, It has been established that Phosphorus-solubilizing bacteria may play a demanding role in increasing the availability of Phosphorus to the crop plants. Although studies are available on summer maize cultivation with reference to planting time but survey of literature reveals that information is lacking on winter maize cultivation.

The present study, keeping the above facts in view was undertaken to find out the Application of Phosphate Solubilizing Bacteria \& its Ecological effect on growth and yield of winter maize (ㄹea mays L.)

\section{Materials And Methods}

The present investigations were carried out on maize (Zea mays hybrid variety all rounder) during the winter seasons of 2011-12 and 2012-13 at Bamin Michi village, Lower Subansiri District Arunachal Pradesh. 
The present field study was laid out in split plot design. The three treatments viz, control $\mathrm{T}_{1}$, seed inoculation with PSB $\mathrm{T}_{2}$ and seed inoculation with PSB along with 5t FYM ha ${ }^{-1} \mathrm{~T}_{3}$ were allotted to plots. Using Fisher and Yates random table (Panse and Sukhatme, 1985) the treatments were allocated to each plot in three replications. There were 9 such plots.

The inter row spacing maintained was $60 \mathrm{~cm}$ with intra row spacing at $25 \mathrm{~cm}$. The observations on growth and yield characteristics of the crop were recorded by using the standard procedures. The data obtained by various observations was subjected to statistical analysis by adopting Method of 'Analysis of Variance' (Cochron and Cox.1992) for determining the significance of difference between the treatment means and to draw valid conclusions.

\section{Results \& Discussion}

\section{Effect Of Treatment On Growth \& Developemental Characters}

In respect to growth parameters, the crop responded absolutely to PSB inoculation. Due to different variables, maize plant population did not have significant variation(Table 4.3). Due to PSB inoculation along with FYM, the values of all growth parameters (plant height(Table 4.2), green leaves (Table 4.3), leaf area(Table 4.4), LAI(Table 4.5) and dry matter production plant ${ }^{-1}$ (Table 4.6)) recorded higher and remained lower under control. However, on number of days to $50 \%$ silking, maturity and barren plants plot ${ }^{-1}$ (Table 4.7), the effect of PSB inoculation alone, PSB added with 5t FYM ha-1 and the control found at par to each other though these characters remained higher and lower with PSB added with FYM and the control correspondingly.

Table: 1 Effect of treatments on initial and final maize plant population plot $^{-1}$

\begin{tabular}{|c|c|c|c|c|}
\hline \multirow{2}{*}{ Treatments } & Initial & \multicolumn{2}{|c|}{ Final } & \\
\hline & $2011 \quad 2012$ & \multicolumn{2}{|r|}{2012} & \\
\hline \multicolumn{5}{|l|}{ PSB } \\
\hline $\mathrm{T}_{1}$ & 67.43 & 67.51 & 63.10 & 62.14 \\
\hline $\mathrm{T}_{2}$ & 67.45 & 67.59 & 63.17 & 62.22 \\
\hline $\mathrm{T}_{3}$ & 67.48 & 67.47 & 63.37 & 62.25 \\
\hline SEm \pm & 0.03 & 0.08 & 0.09 & 0.13 \\
\hline $\mathrm{CD}(\mathrm{P}=0.05)$ & NS & NS & NS & NS \\
\hline
\end{tabular}

Table 4.2 Effect of treatments on plant height $(\mathrm{cm})$

\begin{tabular}{|c|c|c|c|c|c|c|c|c|c|c|}
\hline \multirow[t]{2}{*}{ Treatments } & \multicolumn{2}{|c|}{30 DAS } & \multicolumn{2}{|c|}{60 DAS } & \multicolumn{2}{|l|}{90 DAS } & \multicolumn{2}{|c|}{120 DAS } & \multicolumn{2}{|c|}{ At Harvest } \\
\hline & 2011 & 2012 & 2011 & 2012 & 2011 & 2012 & 2011 & 2012 & 2011 & 2012 \\
\hline PSB & & & & & & & & & & \\
\hline $\mathrm{T}_{1}$ & 08.80 & 09.06 & 32.08 & 29.75 & 115.22 & 114.34 & 215.42 & 211.38 & 225.76 & 218.01 \\
\hline $\begin{array}{l}\mathrm{T}_{3} \\
\mathrm{~T}_{3}\end{array}$ & $\begin{array}{l}09.09 \\
09.63\end{array}$ & $\begin{array}{l}09.23 \\
09.58\end{array}$ & $\begin{array}{l}33.49 \\
35.62\end{array}$ & $\begin{array}{l}30.71 \\
32.25\end{array}$ & $\begin{array}{l}119.87 \\
127.44\end{array}$ & $\begin{array}{l}117.00 \\
122.50\end{array}$ & $\begin{array}{l}219.77 \\
228.29\end{array}$ & $\begin{array}{l}216.01 \\
223.02\end{array}$ & $\begin{array}{l}230.79 \\
238.88\end{array}$ & $\begin{array}{l}222.26 \\
229.58\end{array}$ \\
\hline $\mathrm{SEm} \pm$ & 0.24 & 0.14 & 0.50 & 0.45 & 1.92 & 0.92 & 2.00 & 1.13 & 1.16 & 0.96 \\
\hline $\mathrm{CD}(\mathrm{P}=0.05)$ & NS & NS & 1.47 & 1.33 & 5.60 & 2.69 & 5.85 & 3.31 & 3.39 & 2.80 \\
\hline
\end{tabular}

Table 4.3 Effect of treatments on number of green leaves plant ${ }^{-1}$

\begin{tabular}{|c|c|c|c|c|c|c|c|c|}
\hline \multirow{2}{*}{ Treatments } & \multicolumn{2}{|c|}{$30 \mathrm{DAS}$} & \multicolumn{2}{|c|}{$60 \mathrm{DAS}$} & \multicolumn{2}{|c|}{90 DAS } & \multicolumn{2}{|c|}{$120 \mathrm{DAS}$} \\
\hline & 2011 & 2012 & 2011 & 2012 & 2011 & 2012 & 2011 & 2012 \\
\hline \multicolumn{9}{|l|}{ PSB } \\
\hline $\mathrm{T}_{1}$ & 2.01 & 1.93 & 5.69 & 5.70 & 08.83 & 08.76 & 6.63 & 6.52 \\
\hline $\mathrm{T}_{2}$ & 2.10 & 2.01 & 5.86 & 5.83 & 09.04 & 08.95 & 6.88 & 6.67 \\
\hline $\mathrm{T}_{3}$ & 2.24 & 2.12 & 6.11 & 6.05 & 09.41 & 09.29 & 7.28 & 6.97 \\
\hline SEm \pm & 0.06 & 0.05 & 0.10 & 0.07 & 0.11 & 0.09 & 0.08 & 0.11 \\
\hline $\mathrm{CD}(\mathrm{P}=0.05)$ & NS & NS & 0.28 & 0.22 & 0.33 & 0.25 & 0.22 & 0.33 \\
\hline
\end{tabular}

Table 4.4 Effect of treatments on leaf area plant ${ }^{-1}\left(\mathrm{~cm}^{2}\right)$

\begin{tabular}{|c|c|c|c|c|c|c|c|c|}
\hline \multirow[t]{2}{*}{ Treatments } & \multicolumn{2}{|c|}{30 DAS } & \multicolumn{2}{|c|}{60 DAS } & \multicolumn{2}{|c|}{$90 \mathrm{DAS}$} & \multicolumn{2}{|l|}{$120 \mathrm{DAS}$} \\
\hline & 2011 & 2012 & 2011 & 2012 & 2011 & 2012 & 2011 & 2012 \\
\hline PSB & & & & & & & & \\
\hline $\mathrm{T}_{1}$ & 70.96 & 65.18 & 905.85 & 841.16 & 5352.35 & 5257.47 & 4672.98 & 4650.9 \\
\hline $\mathrm{T}_{2}$ & 72.14 & 66.41 & 936.40 & 892.48 & 5623.45 & 5520.23 & 4901.22 & 4860.9 \\
\hline $\mathrm{T}_{3}$ & 74.56 & 68.38 & 996.16 & 968.45 & 6010.19 & 5963.45 & 5265.34 & 5166.5 \\
\hline SEm \pm & 1.73 & 0.74 & 12.18 & 6.30 & 76.92 & 28.55 & 49.06 & 27.61 \\
\hline $\mathrm{CD}(\mathrm{P}=0.05)$ & NS & 2.15 & 35.55 & 18.38 & 224.48 & 83.33 & 143.19 & 80.57 \\
\hline
\end{tabular}


Table 4.5 Effect of treatments on leaf area index

\begin{tabular}{lllllclcr}
\hline Treatments & \multicolumn{2}{c}{30 DAS } & \multicolumn{3}{c}{ 60 DAS } & \multicolumn{2}{c}{90 DAS } & \multicolumn{2}{c}{ 120 DAS } \\
\cline { 2 - 9 } & 2011 & 2012 & 2011 & 2012 & 2011 & 2012 & 2011 & 2012 \\
\hline PSB & & & & & & & & \\
$\mathrm{T}_{1}$ & 0.047 & 0.043 & 0.58 & 0.54 & 3.55 & 3.49 & 3.10 & 3.08 \\
$\mathrm{~T}_{2}$ & 0.047 & 0.044 & 0.62 & 0.58 & 3.73 & 3.66 & 3.25 & 3.22 \\
$\mathrm{~T}_{3}$ & 0.049 & 0.045 & 0.65 & 0.63 & 4.00 & 3.96 & 3.49 & 3.43 \\
$\mathrm{SEm} \pm$ & 0.001 & 0.0005 & 0.01 & 0.004 & 0.05 & 0.02 & 0.03 & 0.02 \\
$\mathrm{CD}(\mathrm{P}=0.05)$ & $\mathrm{NS}$ & 0.001 & 0.02 & 0.01 & 0.15 & 0.06 & 0.10 & 0.05
\end{tabular}

Table 4.6 Effect of treatments on dry matter accumulation $\left(\mathrm{g} \mathrm{plant}^{-1}\right)$

\begin{tabular}{|c|c|c|c|c|c|c|c|c|c|c|}
\hline \multirow[t]{2}{*}{ Treatments } & \multicolumn{2}{|c|}{$30 \mathrm{DAS}$} & \multicolumn{2}{|c|}{$60 \mathrm{DAS}$} & \multicolumn{2}{|c|}{$90 \mathrm{DAS}$} & \multicolumn{2}{|c|}{$120 \mathrm{DAS}$} & \multicolumn{2}{|c|}{ At Harvest } \\
\hline & 2011 & 2012 & 2011 & 2012 & 2011 & 2012 & 2011 & 2012 & 2011 & 2012 \\
\hline PSB & & & & & & & & & & \\
\hline $\mathrm{T}_{1}$ & 0.24 & 0.23 & 3.80 & 3.78 & 35.53 & 34.44 & 153.84 & 149.20 & 261.61 & 254.43 \\
\hline $\mathrm{T}_{2}$ & 0.25 & 0.23 & 3.97 & 3.93 & 37.70 & 36.21 & 157.14 & 151.88 & 275.20 & 267.29 \\
\hline $\mathrm{T}_{3}$ & 0.26 & 0.24 & 4.22 & 4.14 & 41.24 & 39.22 & 163.57 & 156.44 & 298.07 & 290.19 \\
\hline SEm \pm & 0.01 & 0.005 & 0.07 & 0.05 & 0.73 & 0.08 & 2.08 & 0.57 & 3.74 & 3.62 \\
\hline $\mathrm{CD}(\mathrm{P}=0.05)$ & NS & NS & 0.19 & 0.16 & 2.14 & 0.23 & 6.07 & 1.66 & 10.91 & 10.56 \\
\hline
\end{tabular}

Table 4.7 Effect of treatments on developmental phases and crop lodging

\begin{tabular}{|c|c|c|c|c|c|c|c|c|c|c|}
\hline \multirow[t]{2}{*}{ Treatments } & \multicolumn{2}{|c|}{ Days to $50 \%$ tasseling } & \multicolumn{2}{|c|}{ Days to $50 \%$ silking } & \multicolumn{2}{|c|}{ Days to $50 \%$ maturity } & \multicolumn{2}{|c|}{$\begin{array}{l}\text { Barren plant } \\
\text { plot }^{-1}\end{array}$} & \multicolumn{2}{|c|}{$\begin{array}{l}\text { Crop lodging } \\
\text { (No. of plant plot }^{-1} \text { ) }\end{array}$} \\
\hline & 2011 & 2012 & 2011 & 2012 & 2011 & 2012 & 2011 & 2012 & 2011 & 2012 \\
\hline \multicolumn{11}{|l|}{$\overline{\text { PSB }}$} \\
\hline $\mathrm{T}_{1}$ & 107.28 & 105.05 & 113.36 & 110.32 & 132.07 & 128.35 & 6.98 & 7.40 & 1.20 & 1.20 \\
\hline $\mathrm{T}_{2}$ & 106.82 & 104.57 & 112.69 & 109.87 & 131.36 & 127.68 & 6.51 & 7.17 & 1.09 & 1.09 \\
\hline $\mathrm{T}_{3}$ & 106.30 & 103.91 & 111.48 & 109.17 & 130.33 & 126.77 & 5.72 & 6.54 & 1.42 & 1.37 \\
\hline SEm \pm & 0.78 & 0.54 & 0.43 & 0.22 & 0.43 & 0.36 & 0.14 & 0.14 & 0.12 & 0.10 \\
\hline $\mathrm{CD}(\mathrm{P}=0.05)$ & NS & NS & 1.26 & 0.64 & 1.26 & 1.04 & 0.41 & 0.41 & NS & NS \\
\hline
\end{tabular}

\section{Effect Of Treatments On Yeild And Yeild Attributes}

With the inoculation of PSB added with FYM as compared to PSB alone or the control, the values of almost all the yield attributes as well as grain and stover yields (Table 4.8.1 to 4.10) were recorded maximum.

Table 4.8.1 Effect of treatments on yield attributes

\begin{tabular}{|c|c|c|c|c|c|c|c|c|c|c|c|c|}
\hline \multirow[t]{2}{*}{ Treatments } & \multirow{2}{*}{$\begin{array}{l}\text { Cobs } \\
2011\end{array}$} & $\operatorname{blant}^{-1}$ & \multicolumn{2}{|c|}{ First cobs plot $^{-1}$} & \multicolumn{2}{|c|}{${ }_{1}$ Second cobs plot ${ }^{-}$} & \multicolumn{2}{|c|}{ Cob length $(\mathrm{cm})}$. & \multicolumn{2}{|c|}{ Cob girth $(\mathrm{cm})}$. & \multicolumn{2}{|c|}{ Kernel rowscob } \\
\hline & & 2012 & 2011 & 2012 & 2011 & 2012 & 2011 & 2012 & 2011 & 2012 & 2011 & 2012 \\
\hline \multicolumn{13}{|l|}{ PSB } \\
\hline $\mathrm{T}_{1}$ & 0.87 & 0.85 & 53.21 & 51.85 & 1.77 & 1.52 & 13.10 & 13.06 & 09.73 & 09.62 & 12.17 & 12.13 \\
\hline $\mathrm{T}_{2}$ & 0.88 & 0.86 & 53.73 & 52.12 & 2.00 & 1.97 & 13.41 & 13.38 & 10.00 & 09.89 & 12.32 & 12.27 \\
\hline $\mathrm{T}_{3}$ & 0.90 & 0.89 & 54.72 & 52.78 & 2.57 & 2.66 & 13.96 & 13.91 & 10.42 & 10.29 & 12.55 & 12.51 \\
\hline SEn & 0.009 & 0.003 & 0.24 & 0.24 & 0.07 & 0.05 & 0.11 & 0.10 & 0.15 & 0.16 & 0.13 & 0.12 \\
\hline & 26 & 0.01 & 0.69 & - & 9 & 5 & 33 & 30 & 45 & 0.47 & 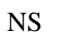 & NS \\
\hline
\end{tabular}

Table 4.8.2 Effect of treatments on yield attributes

\begin{tabular}{|c|c|c|c|c|c|c|c|c|c|c|c|c|}
\hline \multirow[t]{2}{*}{ Treatments } & \multicolumn{2}{|c|}{ row $^{-1}$ Kernels } & \multicolumn{2}{|c|}{ Grains $\mathrm{cob}^{-1}$} & \multicolumn{2}{|c|}{ Test weight (g.) } & \multicolumn{2}{|c|}{ Grain weight $\mathrm{cob}^{-1}$} & \multicolumn{2}{|c|}{$\begin{array}{l}\text { First cobs grain } \\
\text { yield } \\
\left(\mathrm{kg} \mathrm{plot}^{-1}\right)\end{array}$} & \multicolumn{2}{|c|}{$\begin{array}{l}\text { Second cobs } \\
\text { grain } \\
\text { yield (kg } \\
\left.\text { plot }^{-1}\right)\end{array}$} \\
\hline & 2011 & 2012 & 2011 & 2012 & 2011 & 2012 & 2011 & 2012 & 2011 & 2012 & 2011 & 2012 \\
\hline PSB & & & & & & & & & & & & \\
\hline $\mathrm{T}_{1}$ & 26.71 & 26.74 & 395.22 & 393.85 & 233.82 & 232.32 & 93.81 & 92.46 & 4.39 & 4.17 & 0.18 & 0.16 \\
\hline $\mathrm{T}_{2}$ & 27.91 & 27.55 & 415.65 & 408.66 & 238.00 & 235.66 & 99.98 & 97.05 & 4.77 & 4.43 & 0.21 & 0.20 \\
\hline $\mathrm{T}_{3}$ & 29.98 & 28.90 & 451.29 & 434.22 & 247.27 & 242.10 & 111.91 & 105.51 & 5.49 & 4.93 & 0.30 & 0.29 \\
\hline SEm \pm & 0.17 & 0.16 & 4.26 & 3.93 & 2.50 & 1.88 & 1.57 & 1.18 & 0.08 & 0.07 & 0.01 & 0.01 \\
\hline $\mathrm{CD}(\mathrm{P}=0.05)$ & 0.48 & 0.47 & 12.44 & 11.47 & 7.29 & 5.49 & 4.41 & 3.44 & 0.23 & 0.20 & 0.02 & 0.02 \\
\hline
\end{tabular}


Table 4.9 Effect of treatments on shelling percentage and harvest index $(\mathrm{HI})$

Treatments Shelling percentage Harvest index in \%(HI)

$\begin{array}{lllll}2011-2012 & 2012-2013 & 2011-2012 & 2012-2013 & \\ \text { PSB } & & & & \\ \mathrm{T}_{1} & 74.97 & 74.77 & 35.11 & 35.08 \\ \mathrm{~T}_{2} & 75.54 & 75.42 & 35.27 & 35.23 \\ \mathrm{~T}_{3} & 76.85 & 76.78 & 35.56 & 35.42 \\ \mathrm{SEm} \pm & 0.24 & 0.26 & 0.16 & 0.25 \\ \mathrm{CD}(\mathrm{P}=0.05) & 0.71 & 0.77 & \mathrm{NS} & \mathrm{NS}\end{array}$

Table 4.10 Effect of treatments on grain and stover yields $\left(\mathrm{kg} \mathrm{ha}^{-1}\right)$

\begin{tabular}{clllll} 
Treatments & Grain yield & \multicolumn{2}{l}{ Pooled Stover Yield } & & \\
$2011-2012$ & $2012-2013$ & $2011-2012$ & $2012-2013$ & & \\
PSB & & & & & \\
$\mathrm{T}_{1}$ & 5469 & 5229 & 5349 & 9152 & 8772 \\
$\mathrm{~T}_{2}$ & 5873 & 5530 & 5701 & 9786 & 9240 \\
$\mathrm{~T}_{3}$ & 6661 & 6105 & 6383 & 11025 & 10155 \\
$\mathrm{SEm} \pm$ & 92 & 70 & 62 & 169 & 159 \\
$\mathrm{CD}(\mathrm{P}=0.05)$ & 269 & 205 & 182 & 493 & 464
\end{tabular}

\section{Effect Of Treatments On Nutreint Content And Its Uptake And Available Nutreint In Soil}

With the PSB inoculation along with 5t FYM ha ${ }^{-1}$, maximum content of Nitrogen, phosphorus and potassium content in grain and stover, as well as their uptake by grain, stover and total uptake by crop were recorded maximum. With inoculation of PSB along with 5t FYM ha ${ }^{-1}$, the available Nitrogen, phosphorus, and potassium content in soil at crop harvest also remained maximum (Table 4.11 to 4.13).

Table 4.11 Effect of treatments on per cent N, P and K contents in grain and stover

\begin{tabular}{|c|c|c|c|c|c|c|c|c|c|c|c|c|}
\hline \multirow[t]{3}{*}{ Treatments } & \multicolumn{4}{|c|}{$\mathrm{N}$ content } & \multicolumn{4}{|c|}{ P content } & \multicolumn{4}{|c|}{$\mathrm{K}$ content } \\
\hline & \multirow{2}{*}{$\frac{\text { Grain }}{2011}$} & & \multicolumn{2}{|c|}{ Stover } & \multicolumn{2}{|l|}{ Grain } & \multicolumn{2}{|l|}{ Stover } & \multicolumn{2}{|l|}{ Grain } & \multicolumn{2}{|c|}{ Stover } \\
\hline & & 2012 & 2011 & 2012 & 2011 & 2012 & 2011 & 2012 & 2011 & 2012 & 2011 & 2012 \\
\hline \multicolumn{13}{|l|}{ PSB } \\
\hline $\mathrm{T}_{1}$ & 1.60 & 1.59 & 0.43 & 0.43 & 0.31 & 0.30 & 0.18 & 0.17 & 0.40 & 0.39 & 1.61 & 1.63 \\
\hline $\mathrm{T}_{2}$ & 1.61 & 1.60 & 0.44 & 0.44 & 0.32 & 0.30 & 0.19 & 0.18 & 0.40 & 0.40 & 1.62 & 1.63 \\
\hline $\mathrm{T}_{3}$ & 1.63 & 1.62 & 0.44 & 0.44 & 0.33 & 0.31 & 0.20 & 0.19 & 0.41 & 0.40 & 1.63 & 1.64 \\
\hline SEm \pm & 0.01 & 0.01 & 0.01 & 0.004 & 0.003 & 0.003 & 0.003 & 0.002 & 0.01 & 0.005 & 0.01 & 0.004 \\
\hline $\mathrm{CD}(\mathrm{P}=0.05)$ & 0.03 & 0.02 & 0.02 & 0.011 & 0.01 & 0.01 & 0.01 & 0.006 & 0.02 & 0.015 & 0.02 & 0.013 \\
\hline
\end{tabular}

Table 4.12 Effect of treatments on nitrogen and phosphorus uptake by grain and stover and its total uptake (kg $\mathrm{ha}^{-1}$ )

\begin{tabular}{|c|c|c|c|c|c|c|c|c|c|c|c|c|}
\hline \multirow[t]{3}{*}{ Treatments } & \multicolumn{4}{|c|}{$\mathrm{N}$ uptake by } & \multirow[t]{2}{*}{ Total N } & \multirow[t]{2}{*}{ uptake } & \multicolumn{4}{|c|}{ P uptake by } & \multirow[t]{2}{*}{ Total P } & \multirow[t]{2}{*}{ Uptake } \\
\hline & \multicolumn{2}{|l|}{ Grain } & \multicolumn{2}{|l|}{ Stover } & & & \multicolumn{2}{|l|}{ Grain } & \multicolumn{2}{|l|}{ Stover } & & \\
\hline & 2011 & 2012 & 2011 & 2012 & 2011 & 2012 & 2011 & 2012 & 2011 & 2012 & 2011 & 2012 \\
\hline \multicolumn{13}{|l|}{ PSB } \\
\hline $\mathrm{T}_{1}$ & 87.75 & 83.22 & 40.73 & 38.58 & 130.49 & 123.81 & 17.10 & 15.01 & 17.18 & 15.65 & 36.29 & 32.67 \\
\hline $\mathrm{T}_{2}$ & 95.58 & 89.46 & 44.12 & 41.17 & 141.71 & 132.64 & 19.29 & 16.62 & 19.75 & 17.59 & 41.05 & 36.21 \\
\hline $\mathrm{T}_{3}$ & 111.11 & 101.16 & 51.74 & 46.89 & 164.86 & 150.06 & 23.54 & 19.90 & 24.54 & 21.53 & 50.09 & 43.43 \\
\hline SEm \pm & 1.65 & 1.34 & 0.96 & 0.80 & 2.47 & 1.99 & 0.42 & 0.32 & 0.52 & 0.41 & 0.81 & 0.63 \\
\hline $\mathrm{CD}(\mathrm{P}=0.05)$ & 4.81 & 3.90 & 2.80 & 2.35 & 7.15 & 5.82 & 1.23 & 0.93 & 1.53 & 1.19 & 2.35 & 1.85 \\
\hline
\end{tabular}


Table 4.13 Effect of treatments on potassium uptake by grain and stover and its total uptake $\left(\mathrm{kg} \mathrm{ha}^{-1}\right)$

\begin{tabular}{|c|c|c|c|c|c|c|}
\hline \multirow[t]{3}{*}{ Treatments } & \multicolumn{4}{|c|}{ K uptake by } & \multicolumn{2}{|c|}{$\begin{array}{l}\text { Total K } \\
\text { uptake }\end{array}$} \\
\hline & \multicolumn{2}{|l|}{ Grain } & \multicolumn{2}{|l|}{ Stover } & \multirow[b]{2}{*}{2011} & \multirow[b]{2}{*}{2012} \\
\hline & $\overline{2011}$ & 2012 & 2011 & 2012 & & \\
\hline \multicolumn{7}{|l|}{$\overline{\mathrm{PSB}}$} \\
\hline $\mathrm{T}_{1}$ & 22.64 & 20.98 & 149.74 & 144.71 & 174.38 & 167.71 \\
\hline $\mathrm{T}:$ & 24.27 & 22.07 & 160.56 & 152.51 & 186.84 & 176.58 \\
\hline $\mathrm{T}_{3}$ & 29.05 & 25.79 & 183.01 & 169.06 & 214.07 & 196.86 \\
\hline SEm \pm & 0.55 & 0.34 & 3.10 & 2.75 & 3.46 & 3.00 \\
\hline $\mathrm{CD}(\mathrm{P}=0.05)$ & 1.60 & 1.00 & 9.05 & 8.02 & 10.09 & 8.74 \\
\hline
\end{tabular}

\section{Effect Of Treatments On Quality}

In respect of protein and carbohydrates in grain, inoculation on PSB along with 5t FYM ha ${ }^{-1}\left(\mathrm{~T}_{3}\right)$ found superior than inoculation with PSB alone and control (Table 4.14).

Table 4.14 Effect of treatments on percent protein and carbohydrate content in grain

\begin{tabular}{|c|c|c|c|c|c|}
\hline \multirow{2}{*}{ Treatments } & \multicolumn{2}{|c|}{ Protein } & \multicolumn{3}{|c|}{ Carbohydrate } \\
\hline & 2011 & 2012 & & 2011 & 2012 \\
\hline PSB & & & & & \\
\hline $\mathrm{T}_{1}$ & & & 09.01 & & 9.97 \\
\hline $\mathrm{T}_{2}$ & & & 09.19 & & 9.16 \\
\hline $\mathrm{T}_{3}$ & & & 09.49 & & 9.43 \\
\hline $\begin{array}{l}\mathrm{SEm} \pm \\
\mathrm{CD}(\mathrm{P}=0\end{array}$ & & & $\begin{array}{l}0.06 \\
0.17\end{array}$ & & 0.05 \\
\hline
\end{tabular}

Table 4.15 Effect of treatments on available $\mathrm{N}, \mathrm{P}$ and $\mathrm{K}\left(\mathrm{kg} \mathrm{ha}^{-1}\right)$ after crop harvest

\begin{tabular}{|c|c|c|c|c|c|c|}
\hline \multirow[t]{2}{*}{ Treatments } & \multicolumn{2}{|c|}{ Nitrogen } & \multicolumn{2}{|c|}{ Phosphorus } & \multicolumn{2}{|c|}{ Potassium } \\
\hline & 2011 & 2012 & 2011 & 2012 & 2011 & 2012 \\
\hline \multicolumn{7}{|l|}{ PSB } \\
\hline $\mathrm{T}_{1}$ & 210.08 & 211.24 & 15.00 & 14.91 & 182.33 & 183.05 \\
\hline $\mathrm{T}_{2}$ & 210.31 & 211.48 & 15.54 & 15.62 & 182.53 & 183.14 \\
\hline $\mathrm{T}_{3}$ & 214.40 & 216.39 & 16.61 & 16.78 & 184.16 & 185.85 \\
\hline SEm \pm & 0.75 & 0.72 & 0.24 & 0.29 & 1.14 & 1.00 \\
\hline $\mathrm{CD}(\mathrm{P}=0.05)$ & 2.20 & 2.10 & 0.71 & 0.83 & NS & NS \\
\hline
\end{tabular}

\section{Conclusions}

On the basis of above results, the following conclusions may be drawn:-

1. To maximize the yield of winter maize, seeds inoculated with PSB plus 5t FYM ha ${ }^{-1}$ was found best in rice based cropping system under agro-ecological region of Ziro.

2. The winter maize seeds inoculated with PSB plus $5 \mathrm{t} \mathrm{FYM} \mathrm{ha}^{-1}$ produced maximum grain \& Stover yields.

3. Yield of winter maize seeds inoculated with PSB plus 5t FYM ha ${ }^{-1}$ was found most remunerative.

\section{Recommendations}

Winter maize be planted with PSB inoculation plus $5 \mathrm{t} \mathrm{FYM} \mathrm{ha}{ }^{-1}$ for economic grain production.

\section{References}

[1]. Chaudhary, A.R. 1983. Maize in Pakistan, Punjab Agri. Res. Coordination Board, Univ. Agric., Faisalabad.

[2]. Tiwari, K.N. (2001). Phosphorus need of Indian soils and crops. Bett. Crops Int.

[3]. 15(2): 6-10.

[4]. Rai, M. (1998). Wheat and maize in research agenda for crop sciences in country. Indian Fmg. 48(1): 8-15.

[5]. Panse, V.G. and Sukhatme, P.V. (1985). Statistical methods for agricultural workers. ICAR Publication, New Delhi-12. pp. 336340 .

[6]. Cochron, W.G. and Cox, G.M. (1992). Experimental Designs. (2 ${ }^{\text {nd }}$ Ed). John Wiley and Sons, Singapore. Pp53-58. 